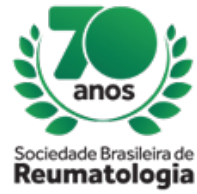

\title{
DISTAL RENAL TUBULAR ACIDOSIS AS INITIAL MANIFESTATION OF SJOGREN'S SYNDROME
}

Leila Patrícia Fonseca Oliveira (Universidade Federal do Ceará, Fortaleza, CE, Brasil), Ruy Sampaio Siqueira Neto (Universidade Federal do Ceará, Fortaleza, CE, Brasil), André Gois Rocha (Universidade Federal do Ceará, Fortaleza, CE, Brasil), Cristiano Nogueira Marques (Universidade Federal do Ceará, Fortaleza, CE, Brasil), Natália Jardim Martins Silva (Universidade Federal do Ceará, Fortaleza, CE, Brasil), Vitor Silva Souza (Universidade Federal do Ceará, Fortaleza, CE, Brasil), Leonardo Ribeiro Sampaio (Universidade Federal do Ceará, Fortaleza, CE, Brasil)

\section{BACKGROUND}

Sjogren's syndrome (SS) is a systemic autoimmune disease that mainly affects lacrimal and salivary glands but can also occur with extraglandular manifestations. We describe a patient with distal renal tubular acidosis (dRTA) manifestations from SS.

\section{CASE REPORT}

A 30-years-old woman presented with fatigue on right leg and muscle weakness for the past 4 months followed by diffuse paralysis and onset calf pain. The patient denied a dry eye and mouth. Physical examination showed a significant decreased muscle strength. Laboratory tests revealed severe hypokalemia, metabolic acidosis, urine alkalinization, serum creatinine was $1.7 \mathrm{mg} / \mathrm{dl}$ and proteinuria 73mg/ 24h, ANA 1: 640 mixed nucleolar and nuclear fine dot pattern, Anti-Ro: reagent, 5mm Shimmer test and urinary $\mathrm{pH}$ 7.0. Salivary glands ultrasound: normal-sized submandibular, irregular contours, heterogeneous echotexture with hypoechoic foci in their parenchyma, more evident to the right, urinary computerized tomography showed ureterolithiasis to the right with mild hydronephrosis and not obstructive nephrolithiasis to the left. The patient was treated with prednisone, sodium bicarbonate and potassium replacement with resolution of hypokalemia and movements improved in 3 days and the renal biopsy was not consent.

\section{CONCLUSION}

Renal involvement occurs in approximately $5 \%$ of SS patients, and dRTA is a consequence of interstitial tubular nephritis responsible for $75 \%$ of these cases. dRTA leads to an inability to acidify urine, potassium loss that can lead to hypocalcemic paresis and chronic acidosis that can cause secondary hyperparathyroidism and hypercalciuria, favoring the renal calculi and nephrocalcinosis. Diagnosis is confirmed with the presence of urinary PH above 5.5 in the presence of serum metabolic acidosis besides the decrease of serum bicarbonate and potassium.

SS is a multisystem autoimmune disease with diagnosis based on the classification criteria. Our patient presented anti-RO/SSA reagent, present in $60-80 \%$ of the cases, shimmer test of $5 \mathrm{~mm}$, confirming the diagnosis. Although salivary gland ultrasound is not included in the classification criteria, is useful for diagnosis increasing the sensitivity. Our patient presented the typical findings of hypoechoic foci.

The treatment for dRTA caused by SS consists on bicarbonate or citrate salts to control acidosis and to avoid renal calculi, potassium replacement. In cases of renal function impairment or significant proteinuria is suggestive to perform renal biopsy and to evaluate treatment with or without associated glucocorticoid to another immunosuppressant. 